\title{
Benefits of Iron supplementation for low birth weight infants: A systematic review
}

\author{
Hui Long, Jing-Mei Yi ${ }^{*}$, Pei-Li Hu, Zhi-Bin Li, Wei-Ya Qiu, Fang Wang and Sing Zhu
}

\begin{abstract}
Background: A number of studies have reported on the effects of iron supplementation in low birth weight infants; however, no systematic review of the available evidence has been conducted to date. Hence, we performed a systematic review of the literature to examine the effects of iron supplementation on hematologic iron status, growth, neurodevelopment, and adverse effects in low birth weight/premature infants.

Methods: We searched the Cochrane Library, Medline, and PubMed for articles reporting on the effects of iron supplementation in low weight infants. The following search terms were used: "preterm born infant(s)/children"; "preterm infants"; "prematurely born children" "weight less than $1500 \mathrm{~g}$ at birth"; "born prematurely"; "low birth weight infant(s)"; "infants born preterm"; "prematurity"; "small-for-gestational age"; "very small gestational age infants"; "iron supplementation"; "iron intake"; "iron supplements"; "ferric and/or ferrous compounds"; and "ferrous sulphate/fumarate/sulfate".

Results: A total of 15 studies were identified and included in the systematic review. Supplemental iron was given orally or as an iron-fortified formula in 14/15 studies. The duration of treatment ranged from 1 week to 18 months. Iron supplementation significantly increased hematologic measures of iron status (including hemoglobin, hematocrit, serum ferritin) relative to placebo or over time in most studies. All controlled studies that examined iron-deficiency anemia (IDA)/ID reported a decreased prevalence of IDA/ID with iron supplementation. Dose dependent decreases in the prevalence of IDA/ID were reported in several studies. Of the 5 studies reporting on growth, none found any significant effect on growth-related parameters (length, height, weight, and head circumference). Only 2 studies reported on neurodevelopment; no marked effects were reported. There were no consistently reported adverse effects, including oxidative stress, inhibited nutrient absorption, morbidity, or the requirement for blood transfusion.
\end{abstract}

Conclusion: The available data suggest that iron supplementation increases the levels of hematologic indicators of iron status and reduces the prevalence of IDA/ID in low birth weight/premature infants. There is insufficient evidence to make a definitive statement regarding the effects of iron supplementation on growth, neurodevelopment, or the occurrence of adverse effects in low birth weight/premature infants.

Keywords: Anemia, Infant, Iron deficiency, Iron supplementation, Low birth weight

\section{Background}

Iron is an essential micronutrient that plays a critical role in many cellular functions and processes, including growth and development. As such, having an adequate supply of iron, along with other micronutrients, is thought to be particularly important for infants [1]. Low birth weight infants, including premature infants, are

\footnotetext{
* Correspondence: YYYijingmei1201@126.com

Department of Pediatrics, General Hospital of Chinese People's Liberation

Army, \#28 Fuxing Road, Haidian District, Beijing 100853, China
}

especially susceptible to developing iron deficiency anemia (IDA) because these infants have smaller iron stores at birth and a greater iron requirement concurrent with a rapid increase in the red cell mass than term infants [2-5]. Increased hemolysis, shortened red blood cell lifespan, low circulating erythropoietin levels, blood sampling, and blood loss associated with medical and surgical procedures may all contribute to anemia in low birth weight and premature infants.

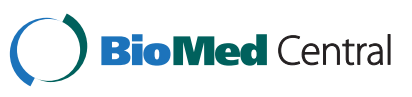

(c) 2012 LONG et al.; licensee BioMed Central Ltd. This is an Open Access article distributed under the terms of the Creative Commons Attribution License (http://creativecommons.org/licenses/by/2.0), which permits unrestricted use, distribution, and reproduction in any medium, provided the original work is properly cited. 
The potential consequences of IDA during infancy include impaired development [6-11] and altered longer term neurodevelopment [12-14]. Due to the high risk of IDA, iron supplementation is recommended for low birth weight infants [15]. Whether or not such supplementation is of any benefit to low birth weight infants is unclear. Interestingly, the findings from several systematic reviews / metaanalyses examining the effects of iron supplementation in children suggest that supplementation improves hematologic markers of iron status [16,17], but does not markedly improve growth, or mental or motor development [18-20]. Although a number of studies have reported on the effects of iron supplementation in low birth weight infants, no systematic review of the available evidence has been conducted to date. Hence, we performed a systematic review of the literature, focusing on the effects of iron supplementation in low birth weight / premature infants on hematologic iron status, growth, and neurodevelopment. We also examined potential adverse effects of iron supplementation.

\section{Methods \\ Search Strategy}

The Cochrane Library, Medline, and PubMed databases were searched from inception to June 2011. We limited the searches to include only articles written in English using the following terms: "preterm born infant(s) / children"; "preterm infants"; "prematurely born children" "weight less than $1500 \mathrm{~g}$ at birth"; "born prematurely"; "low birth weight infant(s)"; "infants born preterm"; "prematurity"; "small-for-gestational age"; "very small gestational age infants"; "iron supplementation"; "iron intake"; "iron supplements"; "ferric and/or ferrous compounds"; and "ferrous sulphate / fumarate / sulfate". Boolean operators (not, and, or) were also used in succession to narrow and widen the search. To locate unpublished materials and avoid systemic bias, we manually searched the references lists of original and review articles for symposia proceedings, poster presentations, and abstract from major pediatric association meetings.

\section{Eligibility Criteria}

Studies were eligible for inclusion if they involved infants who were of low birth weight $(<2500 \mathrm{~g})$ or premature (gestational age $<35$ weeks) and received iron supplementation through the enteral (formula or iron drops) or parenteral routes. Studies involving supplementation of other micronutrients were also eligible for inclusion if the only difference between the treatment and the control groups was iron supplementation.

Studies were excluded if there was no definite length of treatment available, or if supplementation was combined erythropoietin treatment.
The search and inclusion and exclusion criteria were developed by an investigative team, including experts in the field of pediatric development and nutrient research. Our team included a biostatistician who was instrumental in defining the criteria.

\section{Study Selection}

The first step in study selection was the exclusion of duplicates (ie, reports of the same study). The title and abstract of the trials retrieved in the search were scanned and studies that were obviously irrelevant were excluded. The full text of each remaining study was reviewed to establish eligibility and all relevant information and data were extracted.

\section{Data Extraction and Quality Assessment}

Data were extracted by 3 independent reviewers. Each reviewer used a standardized data collection form to increase uniformity and reduce bias. In the case of discrepancy, the reviewers made a consensus decision. The following information / data were extracted from each eligible study: first author; year of publication; journal; eligibility criteria; definition of premature or low-birth weight; number of cases and control; population demographics; hematological data (hemoglobin, hematocrit, serum iron) before iron supplementation; iron supplement dose; duration of iron supplementation; hematological data after iron supplementation; growth status after iron supplementation; and adverse effects of iron supplementation.

The quality of each study identified for inclusion was assessed using the Delphi list for quality assessment of randomized clinical trials [21].

\section{Results}

\section{Literature Search}

A total of 2065 articles were identified by searching the 3 databases and a further 3 articles were identified by bibliographic searches (Figure 1). Of these articles, 3 duplicates were subsequently excluded. Abstracts and titles for the remaining 2065 articles were reviewed and 1962 were subsequently excluded, typically because outcomes of interest were not presented or maternal supplements were given. Full text review led to exclusion of a further 88 articles. Hence, a total of 15 articles [2,22-35] were included in the systematic review.

\section{Study Characteristics}

The studies included in this systematic review were published between 1960 and 2010 and included low birth weight infants aged from birth to 80 days (Table 1). The number of low birth weight infants in the iron supplementation treatment group ranged from 16 to 90 , with most studies having 20 to 40 infants per iron 


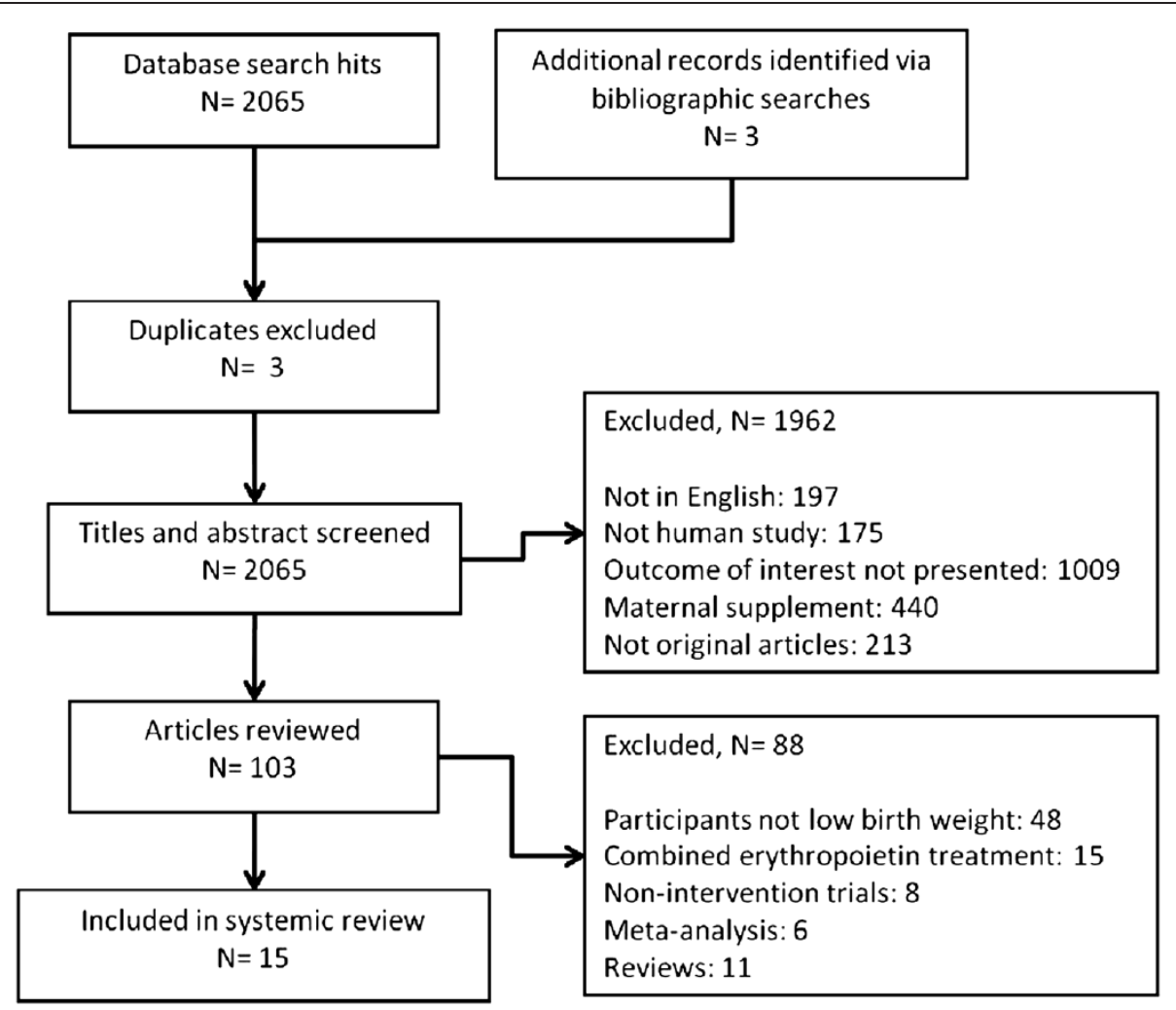

Figure 1 Study flow diagram.

supplementation treatment group. Iron was most commonly given orally $[2,23,27,29-35]$ or as an iron-fortified formula $[2,24-26,28]$. In 1 study, iron was given by intramuscular injection [22]. The duration of iron supplementation varied considerably, ranging from 1 week up to 18 months.

The quality of studies included in the systematic review was generally acceptable for most items of quality assessment. The majority of studies employed a method of randomization $(10 / 15 ; 66.7 \%)$, had similar between group characteristics at baseline $(9 / 15 ; 60 \%)$, specified eligibility criteria $(13 / 15 ; 86.7 \%)$, provided point estimates and measures of variability for the primary outcome measures $(13 / 15 ; 86.7 \%)$, and included an intention-to-treat analysis $(12 / 15 ; 80.0 \%)$. Only $6 / 15$ (40\%) studies provided any definitive information about blinding (outcome assessor, care provider, or patient) (Table 2).

Meta-analyses of the extracted data were not performed because mean values were not available or doses were not adjusted by body weight in the majority of the studies identified. There was significant heterogeneity (data not shown) among the 3 studies in which mean values were available and dose was adjusted for body weight $[31,34,35]$.

\section{Effect of Iron Supplementation on Hematologic Parameters}

All but 1 study reported on the effects of iron supplementation on hematologic measures of iron status (Table 3). The majority of studies reported that iron supplementation significantly increased hematologic measures of iron status relative to control. Specifically, Hammond et al. reported that premature infants treated for 2-4 days with intramuscular iron-dextran $(100 \mathrm{mg})$ had significantly higher hemoglobin concentrations and hematocrit values from 3 months of age onwards and significantly higher erythrocyte counts from 5 months of age onwards compared with premature infants in the control group [22]. In another study, Lundstrom et al. found that low birth weight infants treated for approximately 6 months with an oral iron supplement $(2 \mathrm{mg} / \mathrm{kg} /$ day $)$ had significantly higher hemoglobin, serum ferritin, mean corpuscular volume (MCV), and transferrin saturation levels at 3 months compared with low birth weight infants in the control group [2]. Iwai et al. similarly reported that low birth weight infants fed an iron-fortified formula $(8 \mathrm{mg} / \mathrm{L})$ for 6 months had significantly higher hemoglobin, serum ferritin, and MCV concentrations than control infants fed human milk only [24]. Aggarwal et al. also reported that low birth weight infants fed oral iron in a drop formulation $(3 \mathrm{mg} /$ 
Table 1 Characteristics of the studies examining the effects of iron supplementation in low birth weight infants

\begin{tabular}{|c|c|c|c|c|c|}
\hline Study & $\mathrm{Age}^{\mathrm{a}}$ & $\begin{array}{l}\text { Sample } \\
\text { Size }^{\mathrm{b}}\end{array}$ & Eligibility & $\begin{array}{l}\text { Iron Dose, Duration of Treatment / } \\
\text { Follow-up }\end{array}$ & Measurements \\
\hline $\begin{array}{l}\text { Hammond et al. } \\
\text { 1960[22] }\end{array}$ & $2-3 w k s$ & $\begin{array}{l}\mathrm{T}: 26 \\
\mathrm{C}: 22\end{array}$ & Premature birth & $\begin{array}{l}\text { T: } 100 \text { mg intramuscular iron-dextran; } \\
\text { C: No ironTreatment: } 1 \text { mo; Follow-up: } \\
12 \text { mo }\end{array}$ & Hematologic: Hb, HCT, PCV, RBC \\
\hline $\begin{array}{l}\text { Brozovic et al. } \\
\text { 1974[23] }\end{array}$ & 5 wks & 47 & $\begin{array}{l}\text { GA: } 29-37 \text { wks } \\
\text { BW: } 920-1870 \mathrm{~g}\end{array}$ & $\begin{array}{l}36.3 \mathrm{mg} / \mathrm{d} \text { oral ironTreatment and } \\
\text { follow-up: } 9 \mathrm{mo}\end{array}$ & Hematologic: Hb, SI, TIBC \\
\hline $\begin{array}{l}\text { Lundstrom et al. } \\
\text { 1977[2] }\end{array}$ & 2 wks & $\begin{array}{l}\text { T: } 40 \\
\text { C: } 50\end{array}$ & BW: 1050-2000 g & $\begin{array}{l}\text { T: } 2 \mathrm{mg} / \mathrm{kg} / \mathrm{d} \text { oral iron; C: No } \\
\text { ironTreatment and follow-up: } 6 \text { mo }\end{array}$ & $\begin{array}{l}\text { Hematologic: Hb, MCV, reticulocyte } \\
\text { count, SI, SF, TRNSAT, TIBC }\end{array}$ \\
\hline $\begin{array}{l}\text { Iwai et al. } \\
\text { 1986[24] }\end{array}$ & $3 \mathrm{mo}$ & $\begin{array}{l}\text { Formula: } 30 \\
\text { Human } \\
\text { milk:15 }\end{array}$ & $\begin{array}{l}\text { GA: } 30-40 \text { wks } \\
\text { BW: } 1000-2499 \mathrm{~g}\end{array}$ & $\begin{array}{l}\text { Formula: } 8 \mathrm{mg} / \mathrm{L} \text { oral ironTreatment: } \\
3 \text { mo; Follow-up: } 6 \text { mo }\end{array}$ & $\begin{array}{l}\text { Hematologic: Hb, RBC, SF, iron, TIBC, } \\
\text { MCV }\end{array}$ \\
\hline $\begin{array}{l}\text { Hall et al. } \\
\text { 1993[25] }\end{array}$ & $8-10 d$ & $\begin{array}{l}\text { High iron: } 20 \\
\text { Low iron: } 23 \\
\text { Human } \\
\text { milk: } 13\end{array}$ & $\begin{array}{l}\text { GA: }<35 \text { wks } \\
\text { BW: }<1800 \mathrm{~g}\end{array}$ & $\begin{array}{l}\text { High: } 1.3 \mathrm{mg} / \mathrm{kg} / \mathrm{d} \text { oral iron; Low: } \\
0.3 \mathrm{mg} / \mathrm{kg} / \mathrm{d} \text { oral iron; Milk: } 0.3 \mathrm{mg} / \mathrm{kg} / \mathrm{d} \\
\text { ironTreatment: } 25-34 \text { d; Follow-up: } \sim 11-13 \\
\text { wks }\end{array}$ & $\begin{array}{l}\text { Hematologic: Hb, MCV, TRNSAT, SF, } \\
\text { HCT, RBC, PLFEDevelopment: weight, } \\
\text { length, head circumference }\end{array}$ \\
\hline $\begin{array}{l}\text { Griffin et al. } \\
1999[26]\end{array}$ & $3 d$ & $\begin{array}{l}\text { A: } 29 \\
\text { B: } 34 \\
\text { C: } 15\end{array}$ & $\begin{array}{l}\mathrm{GA}: \geq 32 \mathrm{wks} \\
\mathrm{BW}:<1750 \mathrm{~g}\end{array}$ & $\begin{array}{l}\text { A: } 0.9 \mathrm{mg} / \mathrm{dL} \text { iron formula }(1.17 \mathrm{mg} / \mathrm{kg} / \mathrm{d}) \text {; } \\
\text { B: } 0.5 \mathrm{mg} / \mathrm{dL} \text { iron formula }(0.81 \mathrm{mg} / \mathrm{kg} / \mathrm{d}) ; \\
\text { C: } 0.9 \mathrm{mg} / \mathrm{dL} \text { iron formula until term and } \\
\text { then } 0.5 \mathrm{mg} / \mathrm{dL} \text { iron formula }(0.86 \mathrm{mg} / \mathrm{kg} / \mathrm{d}) \\
\text { Treatment: } 6 \mathrm{mo} \text {; Follow-up: } 12 \mathrm{mo}\end{array}$ & Hematologic: Hb, plasma ferritin \\
\hline $\begin{array}{l}\text { Franz et al. } \\
2000[27]\end{array}$ & $14 / 61 \mathrm{~d}$ & $\begin{array}{l}T: 68 \\
C: 65\end{array}$ & BW: < $1301 \mathrm{~g}$ & $\begin{array}{l}\mathrm{T}: 2-4 \mathrm{mg} / \mathrm{kg} / \mathrm{d} \text { oral iron once enteral } \\
\text { feeding was tolerated; C: No ironTreatment } \\
\text { and follow-up: Until } 61 \text { days of age }\end{array}$ & $\begin{array}{l}\text { Hematologic: SF, TRNAST, HCT, MCV, } \\
M C H, R B C, \text { ID }\end{array}$ \\
\hline $\begin{array}{l}\text { Friel et al. } \\
2001[28]\end{array}$ & Birth & $\begin{array}{l}\text { High: } 29 \\
\text { Normal: } 29\end{array}$ & BW: $<2500 \mathrm{~g}$ & $\begin{array}{l}\text { High: } 20.7 \mathrm{mg} / \mathrm{L} \text { iron formula }(0.6-5.9 \mathrm{mg} / \\
\mathrm{kg} / \mathrm{d}) ; \text { Normal: } 13.4 \mathrm{mg} / \mathrm{L} \text { iron formula (0.6- } \\
3.0 \mathrm{mg} / \mathrm{kg} / \mathrm{d}) \text { Treatment and follow-up: } \\
12 \mathrm{mo}\end{array}$ & $\begin{array}{l}\text { Hematologic: Hb, HCT, SF, TRN, } \\
\text { TRNSAT, MCV, PLFE } \\
\text { Development: WTZ, GDA, HTZ } \\
\text { Oxidative stress: MDA, PLZN, PLCU, } \\
\text { FRAG, CAT, SOD, GHSPX } \\
\text { Adverse effects: frequency of } \\
\text { infection }\end{array}$ \\
\hline $\begin{array}{l}\text { Aggarwal et al. } \\
\text { 2005[29] }\end{array}$ & $50-80 d$ & $\begin{array}{l}\mathrm{T}: 37 \\
\mathrm{C}: 36\end{array}$ & $\begin{array}{l}\mathrm{GA}: \geq 37 \mathrm{wks} \\
\mathrm{BW}:<2500 \mathrm{~g}\end{array}$ & $\begin{array}{l}\text { T: } 3 \text { mg/kg/d oral iron; C: No ironTreatment } \\
\text { and follow-up: } 8 \text { wks }\end{array}$ & $\begin{array}{l}\text { Hematologic: Hb, SF, microcytic } \\
\text { hypochromic, NCHC, NCNC } \\
\text { Development: weight, length, head } \\
\text { circumference }\end{array}$ \\
\hline $\begin{array}{l}\text { Miller et al. } \\
\text { 2006[30] }\end{array}$ & $7-60 d$ & $\begin{array}{l}\text { T: } 16 \\
\text { C: } 16\end{array}$ & GA: 24-32 wks & $\begin{array}{l}\mathrm{T}: 3-12 \mathrm{mg} / \mathrm{kg} / \mathrm{d} \text { oral iron; } \\
\text { C: No ironTreatment and follow-up: } \\
2-3 \text { wks }\end{array}$ & $\begin{array}{l}\text { Hematologic: RBC, SI, SF, TIBC, ZnPP/ } \\
\text { H, sTfRAdverse effects: blood and } \\
\text { urine isoprostanes }\end{array}$ \\
\hline $\begin{array}{l}\text { Arnon et al. } \\
2007[31]\end{array}$ & $\begin{array}{l}2 \text { or } 4 \\
\text { wks }\end{array}$ & $\begin{array}{l}2 \text { wks: } 32 \\
4 \text { wks: } 36\end{array}$ & GA: $<32$ wks & $\begin{array}{l}5 \mathrm{mg} / \mathrm{kg} / \mathrm{d} \text { oral ironTreatment and } \\
\text { follow-up: } 4-6 \text { wks }\end{array}$ & $\begin{array}{l}\text { Hematologic: SI, SF, sTfR, reticulocyte, } \\
\text { HbAdverse effects: morbidity }\end{array}$ \\
\hline $\begin{array}{l}\text { Steinmacher } \\
\text { et al. } \\
\text { 2007[32] }\end{array}$ & $14 / 61 d$ & $\begin{array}{l}\text { Early: } 90 \\
\text { Late: } 74\end{array}$ & BW: < $1301 \mathrm{~g}$ & $\begin{array}{l}\text { Early }(14 \mathrm{~d}): 2-4 \mathrm{mg} / \mathrm{kg} / \mathrm{d} \text { oral iron; } \\
\text { Late }(61 \mathrm{~d}): 2 \mathrm{mg} / \mathrm{kg} / \mathrm{d} \text { oral ironTreatment: } \\
\text { until BW = } 1.6 \times \mathrm{birth} \text { BW; } \\
\text { Follow up: } 5.3 \mathrm{yrs}\end{array}$ & $\begin{array}{l}\text { Neurologic examination, } \\
\text { neurophysiological testing (Gross } \\
\text { Motor Functioning Classification } \\
\text { Scale, } \\
\text { Lincoln-Oseretzky Scale, Kaufmann } \\
\text { Assessment Battery for Children, } \\
\text { visual impairment, and } \\
\text { Child Behavior Check List) }\end{array}$ \\
\hline $\begin{array}{l}\text { Braekke et al. } \\
\text { 2007[33] }\end{array}$ & 5 wks & 21 & $\begin{array}{l}\text { GA: }<32 \text { wksBW: } \\
<1500 \mathrm{~g}\end{array}$ & $\begin{array}{l}9.4 \mathrm{mg} / \mathrm{kg} / \mathrm{d} \text { oral ironTreatment and } \\
\text { follow-up: } 1 \mathrm{wk}\end{array}$ & $\begin{array}{l}\text { Hematologic: Hb, reticulocytes, iron, } \\
\text { SF, TRNSAT } \\
\text { Oxidative stress: urine isoprostane, } \\
\text { urine } 2,3 \text { dinor, plasma total } \\
\text { hydroperoxides } \\
\text { Plasma antioxidants: AA, TAA, DHAA, } \\
\text { alpha tocopherol, FRAP, GGT, total } \\
\text { glutathione }\end{array}$ \\
\hline $\begin{array}{l}\text { Sankar et al. } \\
\text { 2009[34] }\end{array}$ & 2 wks & $\begin{array}{l}\mathrm{T}: 22, \\
\mathrm{C}: 24\end{array}$ & BW: $<1500 \mathrm{~g}$ & $\begin{array}{l}\text { T: } 3-4 \mathrm{mg} / \mathrm{kg} / \mathrm{d} \text { oral iron; } \\
\text { C: No ironTreatment and follow-up: } \\
60 \mathrm{~d}\end{array}$ & $\begin{array}{l}\text { Hematologic: SF, HbAdverse effects: } \\
\text { morbidity }\end{array}$ \\
\hline
\end{tabular}


Table 1 Characteristics of the studies examining the effects of iron supplementation in low birth weight infants (Continued)

\begin{tabular}{|c|c|c|c|c|c|}
\hline \multirow[t]{2}{*}{$\begin{array}{l}\text { Berglund et al. } \\
2010[35]\end{array}$} & 6 wks & $\begin{array}{l}\mathrm{T} 1: 78 \\
\mathrm{~T} 2: 82 \\
\mathrm{C}: 83\end{array}$ & BW: $2000-2500 \mathrm{~g}$ & $\begin{array}{l}\text { T1: } 1 \mathrm{mg} / \mathrm{kg} / \mathrm{d} \text { oral iron; } \\
\text { T2: } 2 \mathrm{mg} / \mathrm{kg} / \mathrm{d} \text { oral iron; } \\
\text { C: No ironTreatment: } 6 \text { wks to } 6 \text { mo of age; } \\
\text { Follow-up: up to } 6 \text { mo }\end{array}$ & $\begin{array}{l}\text { Hematologic: Hb, SF, MCV, PLFE, TRN, } \\
\text { TRNSAT, sTfR, ID, IDA } \\
\text { Development: weight, weight SD } \\
\text { score, length, length SD score, head } \\
\text { circumference, head circumference } \\
\text { SD }\end{array}$ \\
\hline & & & & & $\begin{array}{l}\text { score, knee-heel lengthAdverse } \\
\text { effects: } \\
\text { morbidity }\end{array}$ \\
\hline
\end{tabular}

AA, ascorbic acid; BW, birth weight; C, control; CAT, catalase; CGA, corrected gestational age; d, days; DHAA, dehydroascorbic acid; FRAG, red blood cell fragility; GA, gestational age; GDA, Griffiths' Development Assessment; GHSPx, glutathione peroxidase; GGT, Gamma-glutamyl transferase; Hb, Hemoglobin; HCT,

hematocrit; HTZ, height for age z score; $M C H$, mean corpuscular hemoglobin; MCHC, mean corpuscular hemoglobin concentration; ID, iron deficiency; IDA, iron deficiency anemia; MCV, mean corpuscular volume; MDA, malondialdehyde; mo, month; NA, not available; NCHC, normocytic hypochromic; NCNC, normocytic normochromic; PCV, packed cell volume; PLFE, plasma iron; PLCU, plasma copper; PLZN, plasma zinc; RBC, red blood cell; ROP, retinopathy of prematurity; SF, serum ferritin; SI, serum iron; SOD, superoxide dismutase; STFR, soluble transferrin receptor; T, iron supplementation group; TAA, total ascorbic acid; TIBC, total iron binding capacity; TRN, transferrin; TRNSAT, transferrin saturation; wks, weeks; WTZ, weight for age z score; ZnPP/H, zinc protoporphyrin to heme ratio.

${ }^{a}$ Age at the start of supplementation.

${ }^{b}$ Data analysis sample size.

$\mathrm{kg} /$ day) for 8 weeks had significantly higher adjusted (for age, hemoglobin, ferritin, and maternal hemoglobin) hemoglobin concentrations from 4 weeks onwards compared with control infants [29]. Finally, Berglund et al. found that marginally low birth weight infants fed an oral iron supplement ( 1 or $2 \mathrm{mg} / \mathrm{kg} /$ day) between 6 weeks and 6 months of age had significantly higher hemoglobin, MCV, ferritin, transferrin, iron, transferrin saturation, and transferrin receptor levels compared with control infants [35].

In a non-placebo controlled study, Arnon et al. found that premature infants fed enteral iron $(5 \mathrm{mg} / \mathrm{kg} /$ day $)$ from 2 weeks of age to 8 weeks of age had significantly higher hemoglobin, reticulocyte, iron, and ferritin concentrations compared with premature infants fed enteral iron $(5 \mathrm{mg} / \mathrm{kg} /$ day) from 4 weeks of age to 8 weeks of age [31]. In another non-placebo controlled study, Braekke et al. reported that very low birth weight infants given oral iron supplementation $(9.4 \mathrm{mg} / \mathrm{kg} /$ day $)$ from 6 weeks of age had significantly higher iron and transferrin saturation levels at 7 weeks of age [33].

A small number of studies included in the systematic review found that iron supplementation had no significant effect on hematologic measures of iron status relative to control. These include studies by: Friel et al., in which low birth weight infants were fed either a normal (0.6-3.0 mg/ $\mathrm{kg} /$ day) or high $(0.6-5.9 \mathrm{mg} / \mathrm{kg} /$ day) iron formula from birth for 12 months [28]; Miller et al., in which 7 to 60 day-old premature infants were given oral iron (3 to $12 \mathrm{mg} / \mathrm{kg} /$ day) or control (no iron) for 3 weeks [30]; Sankar et al., in which very low birth weight infants were fed oral iron (3 to $4 \mathrm{mg} / \mathrm{kg} /$ day) or control (no iron) from 2 weeks of age until 60 days of age [34].

A study by Griffin et al. compared the effects of formulations containing either $0.9 \mathrm{mg} / \mathrm{dL}$ iron or $0.5 \mathrm{mg} /$ $\mathrm{dL}$ iron in premature infants and found that there were no dose-dependent differences in hematological indicators of iron status [26]. In another study, Franz et al. reported that early iron supplementation ( 2 to $4 \mathrm{mg} / \mathrm{kg} /$ day once enteral feeding was tolerated) had no significant impact on indicators of iron status in infants with a birth weight $<1301 \mathrm{~g}$ [27].

\section{Effect of Iron Supplementation on the Prevalence of Iron Deficiency and Iron-Deficiency Anemia}

Most studies reported on the prevalence of iron deficiency (ID) and/or IDA (Table 3). All controlled studies that examined the prevalence of ID and/or IDA found that the prevalence of ID and/or IDA was lower in infants who received iron supplementation compared with infants who did not receive iron supplementation $[2,22,24,25,35]$. Several studies also reported dose-dependent effects of iron supplementation on the prevalence of ID and/or IDA, with higher iron doses being associated with decreased prevalence $[25,26,35]$. In contrast, Friel et al. found no obvious effect of dose, with 4/29 (13.8\%) infants in the high iron supplementation group and 2/29 (6.9\%) infants in the normal iron supplementation group having ID after 12 months of treatment [28]. Franz et al. also found that iron supplementation was associated with a decreased prevalence of ID [27].

Only 1 study found no obvious benefit of iron supplementation on the prevalence of ID and/or IDA. Brozovic et al. reported that oral iron $(36.3 \mathrm{mg} /$ day $)$ from 5 weeks to 9 months of age did not prevent ID in premature low birth weight infants [23].

\section{Effect of Iron Supplementation on Growth and Neurodevelopment}

Five studies included in the systematic review examined the effect of iron supplementation on growth, whereas only 2 studies examined the effect of iron supplementation on neurodevelopment (Table 4). None of the studies that examined growth-related variables, including growth rate, length, height, head circumference, and weight, found any effect of iron supplementation 
Table 2 Quality assessment of studies examining the effects of iron supplementation in low birth weight infants

\begin{tabular}{|c|c|c|c|c|c|c|c|c|}
\hline Item & $\begin{array}{l}\text { Was a method of } \\
\text { randomization } \\
\text { used? }\end{array}$ & $\begin{array}{l}\text { Were the groups similar at } \\
\text { baseline regarding the } \\
\text { most important } \\
\text { prognostic indicators? }\end{array}$ & $\begin{array}{l}\text { Were the } \\
\text { eligibility } \\
\text { criteria } \\
\text { specified? }\end{array}$ & $\begin{array}{l}\text { Was the } \\
\text { outcome } \\
\text { assessor } \\
\text { blinded? }\end{array}$ & $\begin{array}{l}\text { Was } \\
\text { the care } \\
\text { provider } \\
\text { blinded? }\end{array}$ & $\begin{array}{l}\text { Was the } \\
\text { patient } \\
\text { blinded? }\end{array}$ & $\begin{array}{l}\text { Were point estimates } \\
\text { and measures of variability } \\
\text { presented for the primary } \\
\text { outcome measures? }\end{array}$ & $\begin{array}{l}\text { Did the analysis } \\
\text { include an } \\
\text { intention-to-treat } \\
\text { analysis? }\end{array}$ \\
\hline $\begin{array}{l}\text { Hammond et al. } \\
\text { 1960[22] }\end{array}$ & $Y$ & $Y$ & NA & NA & NA & NA & $Y$ & $Y$ \\
\hline $\begin{array}{l}\text { Brozovic et al. } \\
\text { 1974[23] }\end{array}$ & NA & NA & Y & NA & NA & NA & Y & $\mathrm{N}$ \\
\hline $\begin{array}{l}\text { Lundstrom et al. } \\
\text { 1977[2] }\end{array}$ & $\mathrm{N}$ & NA & Y & NA & NA & NA & Y & Y \\
\hline Iwai et al. 1986[24] & NA & NA & N & NA & N & $\mathrm{N}$ & Y & Y \\
\hline Hall et al. 1993[25] & Y & Y & Y & NA & NA & Y & Y & Y \\
\hline $\begin{array}{l}\text { Griffin et al. } \\
1999[26]\end{array}$ & Y & $\mathrm{N}$ & Y & NA & NA & Y & N & Y \\
\hline $\begin{array}{l}\text { Franz et al. } \\
2000[27]\end{array}$ & Y & Y & Y & NA & NA & NA & Y & $\mathrm{N}$ \\
\hline $\begin{array}{l}\text { Friel et al. } \\
\text { 2001[28] }\end{array}$ & Y & Y & Y & NA & NA & NA & Y & Y \\
\hline $\begin{array}{l}\text { Aggarwal et al. } \\
\text { 2005[29] }\end{array}$ & Y & Y & Y & NA & NA & Y & Y & Y \\
\hline $\begin{array}{l}\text { Miller et al. } \\
2006[30]\end{array}$ & $\mathrm{N}$ & N & Y & NA & NA & NA & N & Y \\
\hline $\begin{array}{l}\text { Arnon et al. } \\
2007[31]\end{array}$ & Y & Y & Y & NA & NA & NA & Y & $\mathrm{N}$ \\
\hline $\begin{array}{l}\text { Steinmacher et al. } \\
\text { 2007[32] }\end{array}$ & Y & Y & Y & NA & NA & NA & Y & Y \\
\hline $\begin{array}{l}\text { Braekke et al. } \\
\text { 2007[33] }\end{array}$ & $\mathrm{N}$ & NA & Y & NA & NA & NA & Y & Y \\
\hline $\begin{array}{l}\text { Sankar et al. } \\
2009[34]\end{array}$ & Y & Y & Y & Y & N & Y & Y & Y \\
\hline $\begin{array}{l}\text { Berglund et al. } \\
2010[35]\end{array}$ & Y & Y & Y & NA & Y & Y & Y & $Y$ \\
\hline
\end{tabular}

$\mathrm{N}, \mathrm{no}$; NA, information not available or not applicable; $\mathrm{Y}$, yes. 
Table 3 Effects of iron supplementation on hematologic parameters in low birth weight infants

\begin{tabular}{|c|c|c|c|c|c|}
\hline Study & Effect & No Effect & $\begin{array}{l}\% \text { of ID } \\
\text { and/or IDA }\end{array}$ & Conclusion & Comments \\
\hline $\begin{array}{l}\text { Hammond } \\
\text { et al. } \\
1960[22]\end{array}$ & $\begin{array}{l}\mathrm{Hb}, \mathrm{HCT} \text { significantly } \\
\text { higher in T group by } 3 \\
\text { mo; erythrocyte count } \\
\text { significantly higher in } \mathrm{T} \\
\text { group by } 5 \text { mo }\end{array}$ & $\begin{array}{l}\text { Blood volume, } \\
\text { circulating } \mathrm{Hb} \\
\text { mass }\end{array}$ & $\begin{array}{l}27.3 \text { vs } 7.7 \% \\
\text { IDA (C vs T) }\end{array}$ & $\begin{array}{l}\text { Early iron suppl. accelerates } \\
\text { recovery from early IDA }\end{array}$ & $\begin{array}{l}27 \% \text { loss to follow up; other } \\
\text { vitamins were administrated; BW } \\
\text { and hematologic measurements } \\
\text { slightly higher in C vs T group }\end{array}$ \\
\hline $\begin{array}{l}\text { Brozovic et al } \\
1974[23]\end{array}$ & $\begin{array}{l}\text { At } 3 \text { mo of age, most } \\
\text { infants had low serum } \\
\text { iron concentrations, } \\
\text { which remained low }\end{array}$ & NA & $>50 \% \mathrm{IDA}$ & $\begin{array}{l}\text { Iron suppl. was insufficient to } \\
\text { prevent IDA in most infants }\end{array}$ & $\begin{array}{l}\text { No control group; all infants } \\
\text { received vitamin K; some infants } \\
\text { received other vitamins }\end{array}$ \\
\hline
\end{tabular}

$\begin{array}{ll}\text { Lundstrom } & \text { SF, Hb, MCV, TRNSAT } \\ \text { et al. } & \text { significantly higher in T } \\ \text { 1977[2] } & \text { group by } 3 \text { mo }\end{array}$

Reticulocyte $\quad 67$ vs $0 \%$ ID count (C vs T)
Griffin et al. NA

1999[26]
$\mathrm{Hb}, \mathrm{SF}, \mathrm{MCV}$ significantly higher in the formula group by 4 mo

Hall et al 1993[25]

Franz et al. NA 2000[27]

Friel et al. NA

2001[28]

Aggarwal et al. Adjusted $\mathrm{Hb}$ higher in $\mathrm{T}$ 2005[29] group

Miller et al. NA

2006[30]

Arnon et al. 2007[31]

Braekke et al. 2007[33]

Sankar et al. NA 2009[34]
$\mathrm{Hb}$, reticulocytes, iron, ferritin significantly higher in the $2 \mathrm{wk}$ group at $8 \mathrm{wks}$

Iron, TRANSAT

significantly increased
$\mathrm{Hb}, \mathrm{HCT}$, reticulocyte, TRN, TRNSAT

\section{6 vs $33 \%$ ID Breast-fed infants have a high (human milk risk of ID vs formula)}

$27,69,76 \%$ IDA (high, low human milk)

Preterm infants receive more benefit from receiving preterm infant formula containing $1.3 \mathrm{mg} / \mathrm{kg} / \mathrm{d}$ iron vs $0.3 \mathrm{mg} / \mathrm{kg} / \mathrm{d}$
18 vs $27 \%$ ID (A vs B)
0.81-1.17 mg/kg/d iron seems to meet the iron nutritional needs of preterm infants

$\begin{array}{ll}\text { All markers of iron } & 14.7 \text { vs } 40.0 \% \\ \text { nutrition } & \text { ID (T vs C) }\end{array}$

No difference in any hematologic parameters

6.9 vs $13.8 \%$ ID (normal vs high)

SF

NA

SI, SF, TIBC, sTfR NA

Reticulocytes, iron, NA ferritin at 4 wks of age

Ferritin

NA
Fewer infants in group $T$ received blood transfusion vs group C; early iron suppl. is feasible and safe in LBW infants

In terms of cognitive outcome, LBW infants did not benefit from high dose iron

Iron suppl. marginally increases $\mathrm{Hb}$ in LBW infants

Corrected reticulocyte count higher in the $\mathrm{T}$ group suggesting improved erythropoiesis

Iron suppl. to preterm infants as early as 2 weeks of age was more beneficial for iron status, than at 4 weeks of age

Oral iron did not change markers of oxidative stress in LBW infants
$23 \%$ loss to follow up; after 3 mo of age, an increasing number of $C$ group infants were excluded; SF was higher than normal in $\mathrm{C}$ group; iron suppl. given in 2 different forms

Infants in formula group had slightly higher BW than those in human milk group; iron status of LBW infants was not evaluated

44 and $25 \%$ of infants in high and low dose groups dropped out because of prematurity related diseases; $13 \%$ of infants in the human milk group completed the study

In group C, BW was slightly lower, fewer transfusions were received, and $\mathrm{Hb}$ was significantly lower vs $A$ and $B$; no ferritin data for Group C

$34 \%$ loss to follow up; group C tended to have $>$ chronic lung disease and ROP

No control group

$42 \%$ loss to follow up

Iron suppl. was adjusted by individual iron status; CGA, weight, age at enrollment $>$ in $\mathrm{T}$ group

All infants given $25 \mathrm{mg} / \mathrm{d}$ oral vitamin E; $35 \%$ loss to follow up

No control group; all infants received other vitamins, including vitamin E; $15 \%$ loss to follow up; short length of iron administration

Iron suppl. at 2 weeks of age did Iron suppl. group received folic not improve hematological parameters at 2 mo of age in preterm very LBW infants acid and vitamin B12; uncommon iron formulation used 


\section{Table 3 Effects of iron supplementation on hematologic parameters in low birth weight infants (Continued)}

\begin{tabular}{ll}
\hline Berglund et al. & All indicators of iron \\
$2010[35]$ & status differed \\
& significantly between \\
& groups in a \\
& dose-dependent manner
\end{tabular}

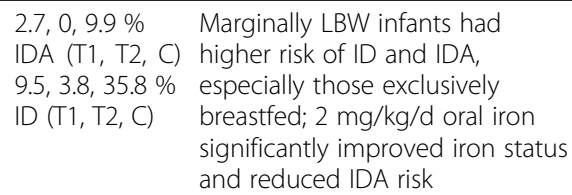

BW, birth weight; C, control; CGA, corrected gestational age; Hb, Hemoglobin; HCT, hematocrit; ID, iron deficiency; IDA, iron deficiency anemia; LBW, low birth weight; MCV, mean corpuscular volume; mo, month; ROP, retinopathy of prematurity; RBC, red blood cell; SF, serum ferritin; SI, serum iron; sTfR, soluble transferrin receptor; suppl., supplementation; T, iron supplementation group; TIBC, total iron binding capacity; TRN, transferrin; TRNSAT, transferrin saturation; wks, weeks; NA, not available.

[25,28,29,34,35]. With regards to neurodevelopment, Friel et al. found that iron supplementation did not improve cognitive development as measured using Griffiths' Development Assessment [28]. In the other study, which focused on examining the effects of iron supplementation on neurocognitive development in premature infants weighing $<1301 \mathrm{~g}$, Steinmacher et al. found that early supplementation ( $<61$ days of age) tended to improve neurocognitive and psychomotor development compared with late supplementation ( $\geq 61$ days of age), as indicated by neurologic examination findings and Gross Motor Function Classification Scores [32].

\section{Adverse Effects of Iron Supplementation}

Several studies included in the systematic review examined potential adverse effects of iron supplementation, including oxidative stress, inhibition of nutrient absorption, neonatal morbidity, the requirement for blood transfusion, and other adverse effects (Table 5). None of the studies found any evidence that iron supplementation increased oxidative stress in low birth weight infants $[28,30,33]$. The study conducted by Friel et al. was the only study to examine absorption of other nutrients with iron supplementation and found that both plasma zinc and copper concentrations were significantly higher for the group of infants who received high vs normal iron supplementation [28]. Three of the 5 studies that reported on neonatal morbidity found no differences between the control and treatment groups or dosedependent effects of iron supplementation on the overall incidence of morbidity [31,34,35]. The other 2 studies found that there was a tendency for there to be a higher prevalence of respiratory tract infection in the iron supplementation group compared with the control group [29] or the high compared with the normal iron supplementation group [28]. Two out of 3 studies examining the need for blood transfusion found no effect of iron supplementation, $[30,34]$ whereas Arnon et al. reported that significantly more infants who started iron supplementation at 4 weeks of age required blood transfusions than infants who started iron supplementation at 2 weeks of age [31]. Another adverse effect reported by Aggarwal et al. included mild vomiting (2/32 infants in the treatment group vs $0 / 30$ infants in the control group) [29].

\section{Discussion}

This is the first systematic review to focus on the effects of iron supplementation in low birth weight / premature infants. Only a relatively small number $(\mathrm{N}=15)$ of studies were identified for review. Several consistent findings were apparent with iron supplementation, including increased concentrations of hematologic indicators of iron status, decreased prevalence of ID / IDA, and a lack of an effect on growth parameters. Few studies that met the criteria for inclusion in our review reported on the effects of iron supplementation on neurodevelopment or the occurrence of adverse effects.

Our observation that iron supplementation generally increased concentrations of hematologic indicators of iron status (notably hemoglobin, hematocrit, and serum ferritin) in low birth weight / premature infants is

Table 4 Effects of iron supplementation on growth and neurodevelopment in low birth weight infants

\begin{tabular}{|c|c|c|}
\hline Study & Anthropometric Data & Neurodevelopmental Outcome \\
\hline Hall et al. 1993[25] & No differences in growth rate, length, head circumference & NA \\
\hline Friel et al. 2001[28] & No differences in WTZ, HTZ at 12 months of age & No difference in GDA; no infant had abnormal development \\
\hline $\begin{array}{l}\text { Steinmacher et al. } \\
2007[32]\end{array}$ & NA & $\begin{array}{l}\text { More infants in the late iron suppl. group had abnormal } \\
\text { neurologic examination; no differences in cognitive } \\
\text { development, mobility, hearing, vision, growth; late vs early } \\
\text { iron suppl. was not an risk factor for abnormal neurologic } \\
\text { examination, disability, or cognitive impairment }\end{array}$ \\
\hline Aggarwal et al. 2005[29] & No differences in weight, length, head circumference & NA \\
\hline Sankar et al. 2009[34] & No difference in weight & NA \\
\hline Berglund et al. 2010[35] & No differences in weight, length, head circumference & NA \\
\hline
\end{tabular}


Table 5 Adverse effects of iron supplementation in low birth weight infants

\begin{tabular}{|c|c|c|c|c|c|}
\hline Study & Oxidative stress & $\begin{array}{l}\text { Inhibition of Other } \\
\text { Nutrient Absorption }\end{array}$ & Neonatal Morbidity & Blood Transfusion & $\begin{array}{l}\text { Other Adverse } \\
\text { Effects }\end{array}$ \\
\hline Hall et al. 1993[25] & NA & $\mathrm{NA}$ & NA & NA & $\begin{array}{l}\text { No adverse effects } \\
\text { in infants who } \\
\text { received higher } \\
\text { iron intake }\end{array}$ \\
\hline Franz et al. 2000[27] & NA & NA & NA & NA & $\begin{array}{l}\text { No adverse effects } \\
\text { once enteral } \\
\text { feeding }(100 \mathrm{~mL} / \\
\mathrm{kg} / \mathrm{d}) \text { tolerated }\end{array}$ \\
\hline Friel et al. 2001 [28] & $\begin{array}{l}\text { No differences in MDA, } \\
\text { SOD, CAT between the } \\
\text { high and normal groups; } \\
\text { GHSPx slightly higher in } \\
\text { the high group }\end{array}$ & $\begin{array}{l}\text { PLCU and PLZN } \\
\text { significantly lower in } \\
\text { high group }\end{array}$ & $\begin{array}{l}\text { Prevalence of respiratory } \\
\text { infection greater in the } \\
\text { high group }\end{array}$ & NA & NA \\
\hline Miller et al. 2006[30] & $\begin{array}{l}\text { No differences in blood } \\
\text { or urine isoprostanes }\end{array}$ & NA & NA & No difference & NA \\
\hline Arnon et al. 2007[31] & NA & NA & No difference & $\begin{array}{l}\text { More transfusions in } \\
\text { the } 4 \text { wk group vs } \\
\text { the } 2 \text { wk group (10/ } \\
36 \text { vs } 1 / 32 \text { ) }\end{array}$ & NA \\
\hline Braekke et al. 2007[33] & $\begin{array}{l}\text { No significant changes in } \\
\text { urine isoprostane, } \\
\text { 2,3-dinor, total } \\
\text { hydroperoxides; plasma } \\
\text { antioxidants were largely } \\
\text { unchanged }\end{array}$ & NA & NA & NA & NA \\
\hline Aggarwal et al. 2005[29] & NA & NA & $\begin{array}{l}\text { Prevalence of respiratory } \\
\text { infection or bronchiolitis } \\
\text { slightly higher in T vs C } \\
\text { group (10/32 vs } 3 / 30)\end{array}$ & NA & $\begin{array}{l}2 \text { infants in the T } \\
\text { group reported } \\
\text { mild vomiting }\end{array}$ \\
\hline Sankar et al. 2009[34] & NA & NA & $\begin{array}{l}\text { No difference ( } 19 \text { vs } 22 \% \\
\text { for T and C groups) }\end{array}$ & $\begin{array}{l}\text { No difference (10 vs } \\
13 \text { for T and C } \\
\text { groups) }\end{array}$ & NA \\
\hline Berglund et al. 2010[35] & NA & NA & No difference & NA & NA \\
\hline
\end{tabular}

C, control; CAT, catalase; GHSPx, glutathione peroxidase; MDA, malondialdehyde; PLCU, plasma copper; PLZN, plasma zinc; T, iron supplementation group; SOD, superoxide dismutase; NA, not available.

consistent with the findings of other systematic reviews / meta-analyses which examined the effects of iron supplementation on hematologic indicators of iron status in children [16] and young children [17]. Unsurprisingly, given the improvements in iron status, there were corresponding decreases in the prevalence of ID/IDA among infants who received iron supplementation. Similar to our review, the findings of several systematic reviews / meta-analyses indicate that iron supplementation does not increase growth in children $[18,19]$. We must emphasize, however, that both the studies included in our review and the previous systematic reviews / meta-analyses typically focused on assessing growth within the first 12 months of supplementation and did not assess longer term growth after iron supplementation. The longer term effect of iron supplementation on growth in low birth weight / premature infants remains to be determined.

As low birth weight / premature infants are particularly vulnerable, and given the fact that humans have no physiologic mechanism for excreting iron, it is important to consider the possibility of iron overload with iron supplementation and identify any potential adverse effects of iron supplementation. Adverse effects of iron supplementation have been suggested, including oxidative stress [36] and an increased risk infection [17]. None of the studies included in our review described any evidence of increased oxidative stress with iron supplementation; however, two studies reported an increased incidence of respiratory tract infection with iron supplementation. Given that iron (and other nutrients) can promote bacterial colonization, an increased risk of respiratory infection is a plausible potential adverse effect of iron supplementation. Further evidence is needed to confirm or deny this possibility. Overall, there were no consistent adverse effects of iron supplementation; however, not all studies monitored for adverse effects of iron supplementation and there was considerable between study variability in the types of adverse effects monitored / reported. Clearly, further studies are on needed on the potential adverse effects of iron supplementation in low birth weight / premature infants. 
This systematic review has a number of limitations that must be acknowledged. Perhaps the most important limitation is was the lack homogeneity between studies in terms of treatment dose, length of treatment, timing of treatment, age of the infants, length of follow-up, and variables assessed. These differences clearly make between study comparisons difficult and almost certainly explain some of the variability in findings. Another limitation is that the length of follow-up was relatively short for the assessment of growth and neurodevelopment. It is possible that any beneficial effects of iron supplementation on development may become apparent many years after supplementation has ceased. Indeed, in the only study with follow-up $>18$ months, Steinmacher et al. found that more infants who received late vs early iron supplementation had abnormal neurologic examination findings at a median of 5.3 years of age [32]. Although a method of randomization was used in most (10/15) studies included in our systematic review, the number of infants included in all studies was relatively small, thus reducing statistical power. Larger scale studies are clearly needed. With regards to the literature search process, we acknowledge the limitation of only considering English language articles for inclusion.

\section{Conclusions}

In conclusion, our review leads us to suggest that iron supplementation can improve indicators of hematologic iron status and reduce the occurrence of ID / IDA in low birth weight / premature infants. The benefits of iron supplementation on growth appear to be limited, at least in the short term (within 18 months of treatment), whereas there is insufficient evidence on the effects of iron supplementation on neurodevelopment or the occurrence of adverse events in this population. Quite clearly larger scale, randomized controlled trials are needed to determine whether iron supplementation in low birth weight / premature infants affects neurodevelopment (short and long term) and long term growth, and to comprehensively monitor potential adverse effects. Such studies should also aim to determine if specific cohorts of low birth weight / premature infants are more likely to benefit from iron supplementation, and the optimal dose, timing, and duration of iron supplementation.

\section{Competing interest}

We have no competing interests to declare.

\section{Authors' contributions}

HL screened studies for inclusion, abstracted data, analyzed data, and drafted the manuscript. JY screened studies for inclusion, abstracted data, analyzed data, and drafted the manuscript. PH screened studies for inclusion, abstracted data, analyzed data, and helped to draft the manuscript. ZL verified outcomes data, assessed study quality, analyzed data, and helped to draft the manuscript. WQ, FW, and SZ critically revised the manuscript for important intellectual content. All authors read and approved the final published manuscript.

\section{Acknowledgements}

Medical writing assistance was provided by MedCom Asia (Taipei, Taiwan).

Received: 16 January 2012 Accepted: 2 July 2012

Published: 16 July 2012

\section{References}

1. Collard KJ: Iron homeostasis in the neonate. Pediatrics 2009, 123:1208-1216.

2. Lundstrom U, Siimes MA, Dallman PR: At what age does iron supplementation become necessary in low-birth-weight infants? J Pediatr 1977, 91:878-883.

3. Rao R, Georgieff MK: Iron therapy for preterm infants. Clin Perinatol 2009, $36: 27-42$.

4. Gorten MK, Cross ER: Iron Metabolism in Premature Infants. li. Prevention of Iron Deficiency. J Pediatr 1964, 64:509-520.

5. Faldella G, Alessandroni R, Salvioli GP, Capelli M, Paolini M, Minak G Jr: Tiraferri S: Lack of correlation between free erythrocyte porphyrin and serum ferritin values at birth and at 2 months of life in low birthweight infants. Arch Dis Child 1983, 58:216-219.

6. Kon N, Tanaka K, Sekigawa M, Negishi Y, Yoshikawa N, Hisata K, Shoji H, Shimizu T: Association between iron status and neurodevelopmental outcomes among VLBW infants. Brain Dev 2010, 32:849-854.

7. Roncagliolo M, Garrido M, Walter T, Peirano P, Lozoff B: Evidence of altered central nervous system development in infants with iron deficiency anemia at 6 mo: delayed maturation of auditory brainstem responses. Am J Clin Nutr 1998, 68:683-690.

8. Lozoff B, Brittenham GM, Viteri FE, Wolf AW, Urrutia JJ: Developmental deficits in iron-deficient infants: effects of age and severity of iron lack. J Pediatr 1982, 101:948-952.

9. Lozoff B, Georgieff MK: Iron deficiency and brain development. Semin Pediatr Neurol 2006, 13:158-165.

10. Shafir T, Angulo-Barroso R, Jing Y, Angelilli ML, Jacobson SW, Lozoff B: Iron deficiency and infant motor development. Early Hum Dev 2008 84:479-485

11. Beard $J$ L: Why iron deficiency is important in infant development. J Nutr 2008, 138:2534-2536.

12. Lozoff B, Jimenez E, Hagen J, Mollen E, Wolf AW: Poorer behavioral and developmental outcome more than 10 years after treatment for iron deficiency in infancy. Pediatrics 2000, 105:E51.

13. Angulo-Kinzler RM, Peirano P, Lin E, Garrido M, Lozoff B: Spontaneous motor activity in human infants with iron-deficiency anemia. Early Hum Dev 2002, 66:67-79.

14. Grantham-McGregor S, Ani C: A review of studies on the effect of iron deficiency on cognitive development in children. J Nutr 2001, 131:649S-666S. discussion 666S-668S.

15. Kleinman RE: Pediatric nutrition handbook. 6th edition. Elk Grove Village, IL: American Academy of Pediatrics; 2009.

16. Gera T, Sachdev HP, Nestel P, Sachdev SS: Effect of iron supplementation on haemoglobin response in children: systematic review of randomised controlled trials. J Pediatr Gastroenterol Nutr 2007, 44:468-486.

17. Iannotti LL, Tielsch JM, Black MM, Black RE: Iron supplementation in early childhood: health benefits and risks. Am J Clin Nutr 2006, 84:1261-1276.

18. Ramakrishnan U, Aburto N, McCabe G, Martorell R: Multimicronutrient interventions but not vitamin a or iron interventions alone improve child growth: results of 3 meta-analyses. J Nutr 2004, 134:2592-2602.

19. Sachdev H, Gera T, Nestel P: Effect of iron supplementation on physical growth in children: systematic review of randomised controlled trials. Public Health Nutr 2006, 9:904-920.

20. Sachdev H, Gera T, Nestel P: Effect of iron supplementation on mental and motor development in children: systematic review of randomised controlled trials. Public Health Nutr 2005, 8:117-132.

21. Verhagen AP, de Vet $H C$, de Bie RA, Kessels AG, Boers $M$, Bouter $L M$, Knipschild PG: The Delphi list: a criteria list for quality assessment of randomized clinical trials for conducting systematic reviews developed by Delphi consensus. J Clin Epidemiol 1998, 51:1235-1241.

22. Hammond D, Murphy A: The influence of exogenous iron on formation of hemoglobin in the premature infant. Pediatrics 1960, 25:362-374. 
23. Brozovic B, Burland WL, Simpson K, Lord J: Iron status of preterm low birthweight infants and their response to oral iron. Arch Dis Child 1974, 49:386-389.

24. Iwai Y, Takanashi T, Nakao Y, Mikawa H: Iron status in low birth weight infants on breast and formula feeding. Eur J Pediatr 1986, 145:63-65.

25. Hall RT, Wheeler RE, Benson J, Harris G, Rippetoe L: Feeding iron-fortified premature formula during initial hospitalization to infants less than 1800 grams birth weight. Pediatrics 1993, 92:409-414.

26. Griffin IJ, Cooke RJ, Reid MM, McCormick KP, Smith JS: Iron nutritional status in preterm infants fed formulas fortified with iron. Arch Dis Child Fetal Neonatal Ed 1999, 81:F45-F49.

27. Franz AR, Mihatsch WA, Sander S, Kron M, Pohlandt F: Prospective randomized trial of early versus late enteral iron supplementation in infants with a birth weight of less than 1301 grams. Pediatrics 2000, 106:700-706.

28. Friel JK, Andrews WL, Aziz K, Kwa PG, Lepage G, L'Abbe MR: A randomized trial of two levels of iron supplementation and developmental outcome in low birth weight infants. J Pediatr 2001, 139:254-260.

29. Aggarwal D, Sachdev HP, Nagpal J, Singh T, Mallika V: Haematological effect of iron supplementation in breast fed term low birth weight infants. Arch Dis Child 2005, 90:26-29.

30. Miller SM, McPherson RJ, Juul SE: Iron sulfate supplementation decreases zinc protoporphyrin to heme ratio in premature infants. J Pediatr 2006, 148:44-48.

31. Arnon S, Shiff Y, Litmanovitz I, Regev RH, Bauer S, Shainkin-Kestenbaum R, Bental Y, Dolfin T: The efficacy and safety of early supplementation of iron polymaltose complex in preterm infants. Am J Perinatol 2007, 24:95-100.

32. Steinmacher J, Pohlandt F, Bode H, Sander S, Kron M, Franz AR: Randomized trial of early versus late enteral iron supplementation in infants with a birth weight of less than 1301 grams: neurocognitive development at 5.3 years' corrected age. Pediatric 2007, 120:538-546.

33. Braekke K, Bechensteen AG, Halvorsen BL, Blomhoff R, Haaland K, Staff AC: Oxidative stress markers and antioxidant status after oral iron supplementation to very low birth weight infants. J Pediatr 2007, 151:23-28.

34. Sankar MJ, Saxena R, Mani K, Agarwal R, Deorari AK, Paul VK: Early iron supplementation in very low birth weight infants-a randomized controlled trial. Acta Paediatr 2009, 98:953-958.

35. Berglund S, Westrup B, Domellof M: Iron supplements reduce the risk of iron deficiency anemia in marginally low birth weight infants. Pediatrics 2010, 126:e874-e883.

36. Thibeault DW: The precarious antioxidant defenses of the preterm infant. Am J Perinatol 2000, 17:167-181.

doi:10.1186/1471-2431-12-99

Cite this article as: Long et al:: Benefits of Iron supplementation for low birth weight infants: A systematic review. BMC Pediatrics 2012 12:99.

\section{Submit your next manuscript to BioMed Central and take full advantage of:}

- Convenient online submission

- Thorough peer review

- No space constraints or color figure charges

- Immediate publication on acceptance

- Inclusion in PubMed, CAS, Scopus and Google Scholar

- Research which is freely available for redistribution

Submit your manuscript at www.biomedcentral.com/submit
( Biomed Central 